\title{
Primary Adenocarcinoma of the Appendix Mimicking Appendicitis: A Case Report
}

\author{
Mohammed Rashid* and Alice Barnes \\ Department of General Surgery, West Suffolk Hospital, Bury St Edmunds, Suffolk, IP332QZ
}

\begin{abstract}
The authors present an unusual case of a 55 year old man who presented with right sided abdominal pain for which he underwent a CT scan. This showed a perforated appendix and he was initially treated in the outpatient setting, before having an appendicectomy, the histology of which showed adenocarcinoma of the appendix. He underwent a right hemicolectomy and adjuvant chemotherapy. He has remained well from his carcinoma point of view, but unfortunately experienced serious side effects from his chemotherapy regime.
\end{abstract}

\section{Introduction}

Primary adenocarcinoma of the appendix is a rare, but important condition. It can mimic appendicitis in its presentation but its identification alters management significantly. To date, only a few hundred cases exist in the literature. Here, the authors present a case of a 55 year old man, who was treated as an outpatient for presumed perforated appendicitis, before being diagnosed with adenocarcinoma of the appendix, which necessitated a right hemicolectomy.

\section{Case}

The patient is a 55 year old male farmer with a 40 year smoking history, a past medical history of hypertension for which he takes Lisinopril $2.5 \mathrm{mg}$ daily. He presented to his GP in January 2011 with right flank pain, which was thought to be renal colic and he underwent an outpatient CT KUB in February 2011. This demonstrated a thickened retrocaecal appendix with an associated inflammatory phlegmon, in keeping with a recent ruptured appendix. On the day of his CT KUB, he was examined by a surgical registrar and was reported to be well, with a soft abdomen and mild right iliac fossa tenderness. His blood tests showed a mild inflammatory response (white cell count 11.3, CRP 119 with otherwise normal U\&Es and LFTs). He was discharged home with 1 week of oral Augmentin $625 \mathrm{mg}$ TDS and a CT scan was repeated in a month's time (March 2011). This showed that the phlegmon around the appendix was now smaller, through the retrocaecal appendix was still thick walled, in keeping with partial resolution of the inflammation (see Figure 1). However, clinically, his right iliac fossa pain continued to repeatedly flare up, and he had three further week long courses of oral Augmentin, which only settled his pain temporarily. He was seen in the General Surgical clinic in April 2011, where he found to be still tender and have a palpable mass in his right iliac fossa and thus he was booked for an appendicectomy the same week. During the operation, a retrocaecal appendix was found but extensive adhesions between the caecum and lateral peritoneal wall necessitated conversion from laparoscopic to open. The operation was otherwise uneventful and the appendix was removed. Histology subsequently came back as a moderately differentiated mucinous adenocarcinoma of the appendix (T4b N0) with clear margins (see Figure 2). A staging CT of the chest and abdomen showed no metastases and in order to excise the lymph node field, he underwent a right hemicolectomy in June 2011, with a side to side stapled anastomosis, after which he recovered well apart from some post operative ileus which settled spontaneously. Following this, it was planned that he would have 8 cycles of adjuvant chemotherapy with Oxaliplatin and Capecitabine, but four hours after having his first dose of Oxaliplatin, he developed acute central crushing chest pain, thought to be due to coronary artery spasm. The Oxaliplatin was substituted for Tegafur, but three weeks after commencing

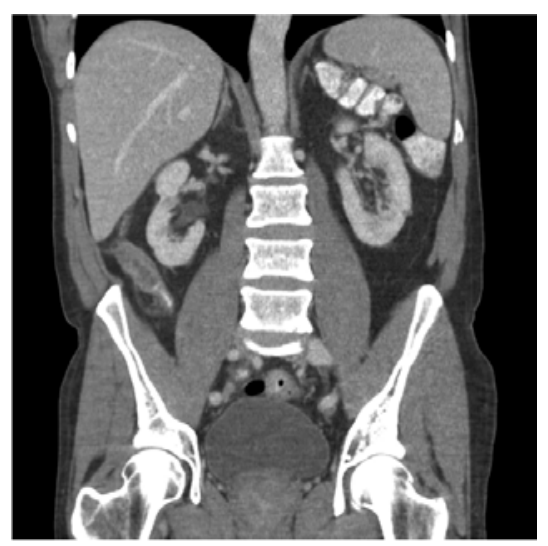

Figure 1: CTAP - A coronal reconstruction view showing a thickened appendix with surrounding abnormal inflammed tissue.

Tegafur, unfortunately he developed enterocolitis, which necessitated hospital admission and treatment with intravenous antibiotics, steroids and fluids. Tegafur was stopped and he is currently waiting to see the Oncologists about the need for ongoing chemotherapy.

\section{Discussion}

The most common primary appendiceal neoplasms are carcinoid tumours, comprising around $32 \%-85 \%$ of all appendiceal tumours $[1,2]$. Primary adenocarcinomas of the appendix, on the other hand, make up only around $4 \%-6 \%$ of all primary appendiceal neoplasms [3] and account for just $0.4 \%-1 \%$ of all gastrointestinal malignancies [4]. The mean age of presentation is in the fifth or sixth decade [5], and the incidence of these neoplasms is 0.12 cases per million people per year [6], making them rare.

Primary adenocarcinomas of the appendix can be of three subtypes: mucinous (55\%), colonic type (34\%) and adenocarcinoid (11\%) [7],

${ }^{*}$ Corresponding author: Mohammed Rashid MRCS, Department of Genera Surgery, West Suffolk Hospital, Bury St Edmunds, Suffolk, IP332QZ, E-mail: amrd02@gmail.com

Received March 22, 2012; Accepted April 12, 2012; Published April 16, 2012

Citation: Rashid M, Barnes A (2012) Primary Adenocarcinoma of the Appendix Mimicking Appendicitis: A Case Report. Surgery Curr Res 2:112. doi:10.4172/21611076.1000112

Copyright: @ 2012 Rashid M, et al. This is an open-access article distributed under the terms of the Creative Commons Attribution License, which permits unrestricted use, distribution, and reproduction in any medium, provided the original author and source are credited. 


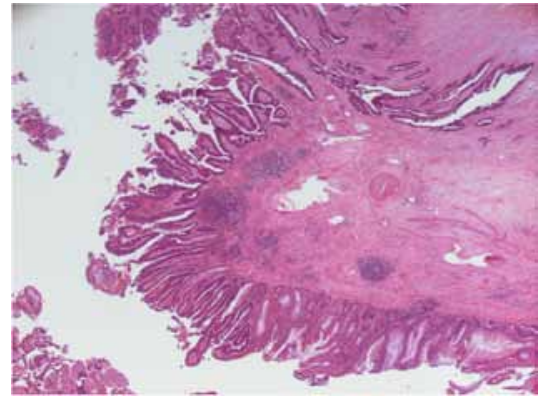

Figure 2: A Haematoxylin and Eosin stained appendix specimen showing a moderately differentiated adenocarcinoma with focal mucin production.

and most neoplasms are only identified after histological examination [8], being found in approximately $0.9 \%-1.4 \%$ of all appendectomy specimens.

Primary adenocarcinomas of the appendix commonly present as acute appendicitis or with a palpable right iliac fossa mass [9], but rarer presentations include urinary frequency mimicking bladder cancer [10], hydronephrosis [11], mimicking Crohn's disease [12], caecal intussusceptions [13] and anaemia [14].

Primary adenocarcinomas of the appendix are the most frequently perforating carcinoma of the gastrointestinal tract [15] and like carcinoma of the colon, spread via local invasion, lymphatic vessels, and the bloodstream to the peritoneal cavity most commonly, followed by lymph nodes, liver, ovaries, the abdominal wall and the lungs [16].

The preferred surgical treatment is still controversial. While simple appendicectomy seems to be sufficient for early, non invasive carcinomas of the appendix [4], most tumours usually present as advanced invasive carcinomas and secondary right hemicolectomy is usually recommended as the operative treatment of choice [17]. The role of adjuvant chemotherapy in adenocarcinomas of the appendix is not clear. Despite this, many oncologists recommend adjuvant 5-fluorouracil based chemotherapy particularly for patients with nodepositive intestinal type adenocarcinoma. Cardiotoxicity and colitis are two rare but serious complications of 5 -fluorouracil therapy $[18,19]$.

\section{References}

1. Connor SJ, Hanna GB, Frizelle FA (1998) Appendiceal tumors: retrospective clinicopathologic analysis of appendiceal tumors from 7,970 appendectomies. Dis Colon Rectum 41: 75-80.
2. Sandor A, Modlin IM (1998) A retrospective analysis of 1570 appendiceal carcinoids. Am J Gastroenterol 93: 422-428.

3. Rutledge RH, Alexander JW (1992) Primary appendiceal malignancies: rare but important. Surgery 111: 244-250.

4. Murphy EM, Farquharson SM, Moran BJ (2006) Management of an unexpected appendiceal neoplasm. Br J Surg 93: 783-792.

5. Chen KT, Spaulding RW (1991) Appendiceal carcinoma masquerading as primary bladder carcinoma. J Urol 145: 821-822.

6. McGory ML, Maggard MA, Kang H, O'Connell JB, Ko CY (2005) Malignancies of the appendix: beyond case series reports. Dis Colon Rectum 48: 2264-2271.

7. Ruoff C, Hanna L, Zhi W, Shahzad G, Gotlieb V, et al. (2011) Cancers of the Appendix: review of the Literatures. ISRN Oncol 2011: 728579.

8. Aljarabah MM, Borley NR, Wheeler JM (2007) Appendiceal adenocarcinoma presenting as left-sided large bowel obstruction, a case report and literature review. Int Semin Surg Oncol 4: 20.

9. Guraya SY, Almaramhy HH (2011) Clinicopathological features and the outcome of surgical management for adenocarcinoma of the appendix. World J Gastrointest Surg 3: 7-12

10. Baskin LS, Stoller ML (1991) Unusual appendiceal pathology presenting as urologic disease. Urology 38: 432-436.

11. Smith JW, Kemeny N, Caldwell C, Banner P, Sigurdson E, et al. (1992) Pseudomyxoma peritonei of appendiceal origin. The Memorial Sloan-Kettering Cancer Center experience. Cancer 70: 396-401.

12. Young RH, Gilks CB, Scully RE (1991) Mucinous tumors of the appendix associated with mucinous tumors of the ovary and pseudomyxoma peritonei. A clinicopathological analysis of 22 cases supporting an origin in the appendix. Am J Surg Pathol 15: 415-429.

13. Lee CT, Lien WC, Wang HP, Lin BR, Huang PH, et al. (2006) Primary appendiceal adenocarcinoma with cecocolic intussusception. J Gastroenterol Hepatol 21: 1079-1081.

14. Yamada T, Murao Y, Nakamura T, Tabuse H, Miyamoto S, et al. (1997) Primary adenocarcinoma of appendix, colonic type associated with perforating peritonitis in an elderly patient. J Gastroenterol 32: 658-662.

15. Cerame MA (1988) A 25-year review of adenocarcinoma of the appendix. A frequently perforating carcinoma. Dis Colon Rectum 31:145-150.

16. Ozakyol AH, Sariçam T, Kabukçuoğlu S, Cağa T, Erenoğlu E (1999) Primary appendiceal adenocarcinoma. Am J Clin Oncol 22: 458-459.

17. Cortina R, McCormick J, Kolm P, Perry RR (1995) Management and prognosis of adenocarcinoma of the appendix. Dis Colon Rectum 38: 848-852.

18. Akay S, Ozütemiz O, Doganavsargil B (2004) Severe colitis after administration of UFT chemotherapy for temporal bone carcinoma. Expert Opin Drug Saf 3 89-92.

19. Dalzell J, Samuel LM (2009) The spectrum of 5-fluorouracil cardiotoxicity Anticancer Drugs 20: 79-80. 